\title{
AVALIAÇÃO SENSORIAL DE BISCOITO SEM GLÚTEN DO TIPO COOKIE DESENVOLVIDO COM FARINHA DE AMARANTO E ENRIQUECIDO COM FARINHA DE BARU
}

Sensory evaluation of cookie gluten-free cookie developed with amaranth and baru flours

Evaluación sensorial de cookies sin gluten desarrolladas con harina de amaranth y baru

Erika Suellen dos Santos Vieira ${ }^{* 1,2}$, Lara Milhomem Guida², Abraham Damian Giraldo Zuniga $^{2}$, Caroline Roberta Freitas Pires ${ }^{3}$

${ }^{1}$ Acadêmica do Curso de Engenharia de Alimentos, Universidade Federal do Tocantins, Palmas -TO, Brasil.

${ }^{2}$ Laboratório de Processos de Separação de Biomoléculas e Desidratação, Programa de Pós-Graduação em Ciência e Tecnologia de Alimentos (PPGTA), Universidade Federal do Tocantins, Palmas -TO, Brasil.

${ }^{3}$ Laboratório de Tecnologia de Alimentos, Programa de Pós-Graduação em Ciência e Tecnologia de Alimentos (PPGTA), Universidade Federal do Tocantins, Palmas -TO, Brasil.

*Correspondência: Laboratório de Processos de Separação de Biomoléculas e Desidratação, Universidade Federal do Tocantins, Av. NS 15, 109 Norte, Palmas, Tocantins, Brasil. CEP:77.010-090. erikasuellen@uft.edu.br.

\section{RESUMO}

Indivíduos celíacos quando expostos ao glúten, sofrem um processo inflamatório que envolve a mucosa do intestino delgado, levando à atrofia das vilosidades intestinais. A utilização de farinhas sem glúten como a de amaranto e a de baru no desenvolvimento de novos produtos de panificação é uma alternativa para contornar a baixa disponibilidade e alto custo de produtos alimentícios sem glúten no mercado. O objetivo deste trabalho foi avaliar a aceitação sensorial de biscoito sem glúten do tipo cookie desenvolvido com farinha de amaranto e enriquecido com diferentes concentrações de farinha de baru. Para o cookie enriquecido com 40 gramas de baru, 70\% dos provadores atribuíram notas entre 6 a 9 para os atributos aroma, textura, impressão global e aparência, valores estes correspondendo entre os termos "gostei ligeiramente" e "gostei muitíssimo", e aproximadamente $46 \%$ dos provadores afirmaram que provavelmente e/ou certamente comprariam. O preço unitário de $10 \mathrm{~g}$ de biscoito, variou entre $\mathrm{R} \$ 0,15$ e $\mathrm{R} \$ 0,16$, sendo possível afirmar preliminarmente que o produto está dentro do valor de mercado. O biscoito sem glúten do tipo cookie desenvolvido com farinha de amaranto e enriquecido com farinha de baru se mostrou uma excelente alternativa de consumo para o público celíaco.

Palavras-chave: cookies; amaranto; baru.

\section{ABSTRACT}

Celiac individuals when exposed to gluten undergo an inflammatory process involving the small intestine mucosa, leading to atrophy of the intestinal villi. The use of gluten-free flour such as amaranth and baru in the development of new bakery products is an alternative to circumvent the low availability and high cost of gluten-free food products on the market, as well as having an excellent nutrient content. The objective of this work was to evaluate the sensory acceptance of a gluten-free cookie biscuit developed with amaranth flour and enriched with different concentrations 
of baru flour. For the cookie enriched with 40 grams of baru, 70\% of the tasters scored between 6 and 9 for the attributes aroma, texture, overall impression and appearance, values corresponding to the terms "slightly liked" and "very liked", and approximately 46\% of tasters said they would probably and / or would certainly buy. The unit price of $10 \mathrm{~g}$ of biscuits ranged from $R \$ 0.15$ to $R \$ 0.16$, and it can be preliminarily stated that the product is within market value. The gluten-free cookie type cookie developed with amaranth flour and enriched with baru flour proved to be an excellent consumption alternative for the celiac audience.

Keywords: cookies; amaranth; baru.

\section{RESUMEN}

Las personas celíacas cuando se exponen al gluten se someten a un proceso inflamatorio que involucra la mucosa del intestino delgado, lo que conduce a la atrofia de las vellosidades intestinales. El uso de harina sin gluten como el amaranto y el barú en el desarrollo de nuevos productos de panadería es una alternativa para evitar la baja disponibilidad y el alto costo de los productos alimenticios sin gluten en el mercado. El objetivo de este trabajo fue evaluar la aceptación sensorial de una galleta de galletas sin gluten desarrollada con harina de amaranto y enriquecida con diferentes concentraciones de harina de baru. Para la galleta enriquecida con 40 gramos de baru, el 70\% de los catadores obtuvieron una puntuación entre 6 y 9 para los atributos aroma, textura, impresión general y apariencia, valores correspondientes a los términos "ligeramente gustadas" y "muy gustadas", y aproximadamente $46 \%$ de los catadores dijeron que probablemente comprarían y/o comprarían. El precio unitario de $10 \mathrm{~g}$ de galletas osciló entre $R \$ 0,15$ y $R \$ 0,16$, y se puede afirmar preliminarmente que el producto está dentro del valor de mercado. La galleta desarrollada demostró ser una excelente alternativa de consumo para el público celíaco.

Palabras clave: cookies; amaranto, baru.

\section{INTRODUÇÃO}

A doença celíaca é uma enteropatia crônica do intestino delgado, de caráter autoimune, onde indivíduos propensos geneticamente quando em contato direto ou indireto ao glúten, sofrem um processo inflamatório que envolve a mucosa do intestino delgado, levando à atrofia das vilosidades intestinais (BRASIL, 2015).

O tratamento da doença celíaca é principalmente através de dietoterapia. Nela é realizado a exclusão do glúten, que é um componente proteico encontrado no trigo, centeio, cevada, aveia e derivados. Para garantir uma dieta livre de glúten, o celíaco deve ter uma atenção rigorosa em relação aos ingredientes das preparações alimentares que serão consumidas, e criar o hábito de sempre fazer a leitura dos rótulos de produtos industrializados. (ARAÚJO et al., 2010).

Nos últimos anos, para driblar a baixa disponibilidade e limitação dos produtos destinados à celíacos, ingredientes de baixo custo passaram a ser estudados como alternativas no desenvolvimento de novos produtos, tais como as farinhas de arroz, soja, coco e de amaranto. (QUEIROZ et al., 2017; MARIANI et al., 2015; CAPRILES et al, 2008)

O amaranto (Amaranthus spp.) é uma planta de alto valor nutritivo e proteico. Possui grãos que podem ser triturados formando uma farinha que é indicada para a elaboração de produtos para celíacos, visto que não possui glúten e substitui outras farinhas como a de trigo, mantendo uma elasticidade e viscosidade semelhante no alimento. (CABRERA-CHAVEZ et al., 2012; CAPRILES et al, 2008)

As características nutricionais dos grãos de amaranto apresentam percentual proteico entre 12 a $17 \%$, com perfil de aminoácidos balanceado com alta quantidade de lisina, apresentando valor nutricional 
semelhante ao leite, à carne e ao ovo (COSTA et al, 2005).

A crescente demanda por alimentos benéficos à saúde é acompanhada pela busca de novos produtos e pela exploração de matérias-primas pouco exploradas, como o baru (Dipteryx alata), planta cujo fruto é uma leguminosa e a amêndoa pode ser consumida crua ou torrada, apresentando grande potencial para utilização em preparações e enriquecimento de produtos alimentícios como doces, bolos, biscoitos e paçocas (ORTOLAN et al, 2016).

A farinha de baru pode ser utilizada nas preparações de produtos para indivíduos celíacos, por ser desprovido de glúten. Possui quantidades consideráveis de carboidrato, lipídeos e proteínas, além de ser rica em micronutriente como cálcio, fósforo e manganês. Apresenta também quantidades relevantes de compostos bioativos, como fenólicos totais e boa capacidade antioxidante (OLIVEIRA et al, 2018)

Um dos problemas enfrentados diariamente pelo público celíaco é a baixa disponibilidade e alto custo de produtos alimentícios sem glúten no mercado. A utilização de farinhas sem glúten como a de amaranto e a de baru é uma alternativa para desenvolvimento de novos produtos de panificação, além de apresentarem um excelente conteúdo de nutrientes. Diante disso, o objetivo deste trabalho foi avaliar a aceitação sensorial de biscoito sem glúten do tipo cookie desenvolvido com farinha de amaranto e enriquecido com diferentes concentrações de farinha de baru.

\section{MATERIAIS E MÉTODOS}

\section{Matéria-Prima}

Foram utilizados os seguintes ingredientes para a formulação do cookie: Açúcar mascavo, bicarbonato de sódio, farinha de amaranto, farinha de baru, fermento químico em pó, gordura vegetal, leite e ovos.
Os frutos do baru foram comprados já torrados e despeliculados em uma casa de produtos naturais em Palmas -TO, onde também foram adquiridos a farinha de amaranto e o açúcar mascavo. Os demais ingredientes empregados na formulação foram adquiridos no mercado local da cidade. Para todos os produtos adquiridos observou-se a data de validade e integridade das embalagens. O baru foi levado ao Laboratório de Processos de Separação de Biomoléculas e Desidratação (LAPSDEA) da Universidade federal do Tocantins, onde foi cortado em pequenas partes e triturado para obtenção da farinha em um liquidificador doméstico.

\section{Elaboração dos biscoitos tipo cookie}

Foram realizados ensaios preliminares para definir a formulação da massa dos diferentes tratamentos experimentais adaptando a metodologia de Júnior et al. (2007), de forma que os ingredientes foram padronizados, variando apenas a proporção de farinha de baru. Os ingredientes utilizados na formulação do biscoito tipo cookie e suas respectivas proporções estão apresentados na Tabela 1 .

Tabela 1. Ingredientes utilizados na formulação do biscoito tipo cookie com diferentes proporções de farinha de baru.

\begin{tabular}{cccc}
\hline & \multicolumn{3}{c}{ Formulação } \\
\hline Ingrediente & FI (g) & FII (g) & FII (g) \\
\hline Açúcar mascavo & 133,3 & 133,3 & 133,3 \\
Bicarbonato de sódio & 10 & 10 & 10 \\
Farinha de amaranto & 333,3 & 333,3 & 333,3 \\
Farinha de Baru & 40 & 80 & 120 \\
Fermento químico em pó & 14 & 14 & 14 \\
Gordura Vegetal & 166,67 & 166,67 & 166,67 \\
Leite & 17,2 & 17,2 & 17,2 \\
Ovos & 140 & 140 & 140 \\
\hline
\end{tabular}

Os ingredientes de cada formulação foram pesados, e em seguida, transferidos para recipientes 
grandes, onde cada massa foi misturada manualmente até a obtenção de um creme homogêneo. Em seguida, os biscoitos foram moldados em formato de cookie com auxílio de uma colher de chá, cada massa tendo entre 12 a 15 g cada e, em seguida, depositados em forma de alumínio retangular, previamente forrada com papel manteiga. Os biscoitos foram assados por quinze minutos em forno elétrico, pré-aquecido a $180^{\circ} \mathrm{C}$. Após assados, os biscoitos foram resfriados à temperatura ambiente e acondicionados em sacos de polietileno identificados.

As formulações tiveram seus nutrientes calculados quantitativamente, a partir da base de dados da Tabela Brasileira de Composição de Alimentos TACO (LIMA, 2006), com os valores diários baseados em uma dieta de $2.000 \mathrm{kcal}(8400 \mathrm{~kJ})$, sendo recomendado o consumo de uma porção de $30 \mathrm{~g}$ (3 unidades).

Os custos de cada formulação, rendimento e custos por porção foi determinado a partir da elaboração da ficha técnica de preparo. Por se tratar de um estudo preliminar, não se levou em consideração os custos com água, gás, energia elétrica, funcionários, impostos, embalagens e depreciação dos equipamentos, apenas os valores das matérias-primas adquiridas no comércio local de Palmas/TO.

\section{Análise Sensorial}

O trabalho foi submetido ao Comitê de Ética visando garantir a obediência à Resolução No 466/12, de 12 de dezembro de 2012 (BRASIL, 2013). A sessão foi conduzida apresentando-se três amostras de biscoito tipo cookie tendo em média $10 \mathrm{~g}$ cada, correspondentes às três diferentes formulações contendo concentrações variadas de farinha de baru, apresentadas aos provadores à temperatura de consumo, com códigos de três dígitos. A ordem de apresentação foi balanceada e aleatória pelo delineamento de uma tabela de números. A avaliação sensorial foi realizada por uma equipe de 61 provadores não treinados, acadêmicos dos cursos da Universidade Federal do Tocantins, de ambos os sexos e idades entre 18 e 40 anos. No teste de aceitabilidade dos biscoitos foram avaliados os atributos de cor, aroma, textura e impressão global utilizando escala hedônica estruturada de nove pontos que abrangiam desde desgostei muitíssimo à gostei muitíssimo, conforme descreve Chaves (2002). Para avaliar a intenção de compra do produto utilizou-se uma escala de cinco pontos, ancorado nos extremos pelos termos: certamente não compraria à certamente compraria. Um teste de ordenação foi utilizado para avaliar a preferência, onde os provadores foram orientados a ordenar em ordem crescente as amostras de acordo com a sua preferência (SILVA, 2016).

\section{Análise dos Dados}

Os dados obtidos foram avaliados por meio do teste de ordenação de Friedman e pelo método de análise de variância (ANOVA) com comparação de médias pelo teste de Tukey, por meio do programa estatístico SISVAR (FERREIRA, 2011). A composição nutricional foi comparada com os valores encontrados na literatura e os custos da formulação foram comparados com cinco tipos de biscoitos tipo cookie sem glúten de marcas variadas encontradas à venda atualmente em casas de produtos naturais e supermercados de Palmas -TO.

\section{RESULTADOS E DISCUSSÃO}

\section{Análise Sensorial}

Na Tabela 2 estão expressos os valores médios das notas obtidas durante a análise sensorial. 
Tabela 2. Valores médios das notas para cada atributo obtidas com análise sensorial das amostras de biscoito tipo cookie.

\begin{tabular}{cccccc|}
\hline Cookie & Aroma & Sabor & Textura & $\begin{array}{c}\text { Impressão } \\
\text { Global }\end{array}$ & Aparência \\
\hline $\begin{array}{c}\text { Enriquecido com } \\
40 \text { gramas de Baru }\end{array}$ & $6,95 \mathrm{a}$ & $7,24 \mathrm{a}$ & $7,01 \mathrm{a}$ & $7,19 \mathrm{a}$ & $7,19 \mathrm{a}$ \\
$\begin{array}{c}\text { Enriquecido com } \\
80 \text { gramas de Baru }\end{array}$ & $6,92 \mathrm{a}$ & $6,11 \mathrm{~b}$ & $5,93 \mathrm{~b}$ & $6,13 \mathrm{~b}$ & $5,95 \mathrm{~b}$ \\
$\begin{array}{c}\text { Enriquecido com } \\
120 \text { gramas de } \\
\text { Baru }\end{array}$ & $6,75 \mathrm{a}$ & $6,27 \mathrm{~b}$ & $6,13 \mathrm{~b}$ & $6,15 \mathrm{~b}$ & $6,39 \mathrm{ab}$ \\
\hline
\end{tabular}

Letras iguais na mesma coluna não diferem significativamente $(\mathrm{p}<0,05)$.

De acordo com os resultados expressos na tabela, observa-se que para o atributo aroma não houve diferença significativa entre as três formulações de cookie tendo seus valores situados na escala entre os termos "gostei ligeiramente" e "gostei moderadamente". Para os atributos sabor, textura e impressão global o cookie enriquecido com 40 gramas de farinha de baru obteve as maiores médias de aceitação se diferindo ao nível de 5\% de significância pelo Teste de Tukey do cookie enriquecido com 80 e 120 gramas de farinha de baru. Entretanto, entre as amostras estudadas não houve diferença significativa entre o cookie formulado com 40 gramas de farinha de baru em comparação com o cookie formulado com 120 gramas da mesma farinha. $\mathrm{O}$ cookie enriquecido com 40 gramas de baru possuiu resultados excelentes, tendo em vista que o produto foi indicado pela maioria dos provadores entre "gostei moderadamente" e "gostei muito" em quatro dos cincos atributos julgados acima.

Para os atributos aroma, textura, impressão global e aparência (Figura 1) observa-se que mais de $70 \%$ dos provadores atribuíram notas entre 6 a 9 , valores estes correspondendo entre os termos "gostei ligeiramente" e "gostei muitíssimo" do cookie enriquecido com 40 gramas de farinha de baru.

Júnior et al. (2007) ao determinar a qualidade de biscoitos formulados com diferentes concentrações de baru em relação ao atributo sabor, observaram que não houve diferença significativa quanto à preferência entre a amostra controle e os demais tratamentos. No entanto, os biscoitos com $2 \%$ e $6 \%$ de farinha de amêndoa de baru foram significativamente as mais preferidas em relação ao tratamento com $8 \%$ da mesma farinha.

Outros autores obtiveram biscoitos tipo cookie de boa qualidade tecnológica e com bom nível de aceitação provenientes de outros frutos como é o caso do jatobá. Em face dessa boa aceitação, os autores acreditaram ser possível o aumento da substituição de farinha de trigo por farinha de jatobá ou baru na fórmula, em níveis superiores a $10 \%$, sem perdas da qualidade sensorial do produto final. Essa porcentagem de $10 \%$ se enquadra na formulação do cookie elaborado com 40 gramas de farinha de baru, visto que a massa total representava cerca de 430 gramas. 
Figura 1. Histogramas de frequência quanto à avaliação sensorial de biscoitos tipo cookie enriquecido com diferentes concentrações de farinha de baru.

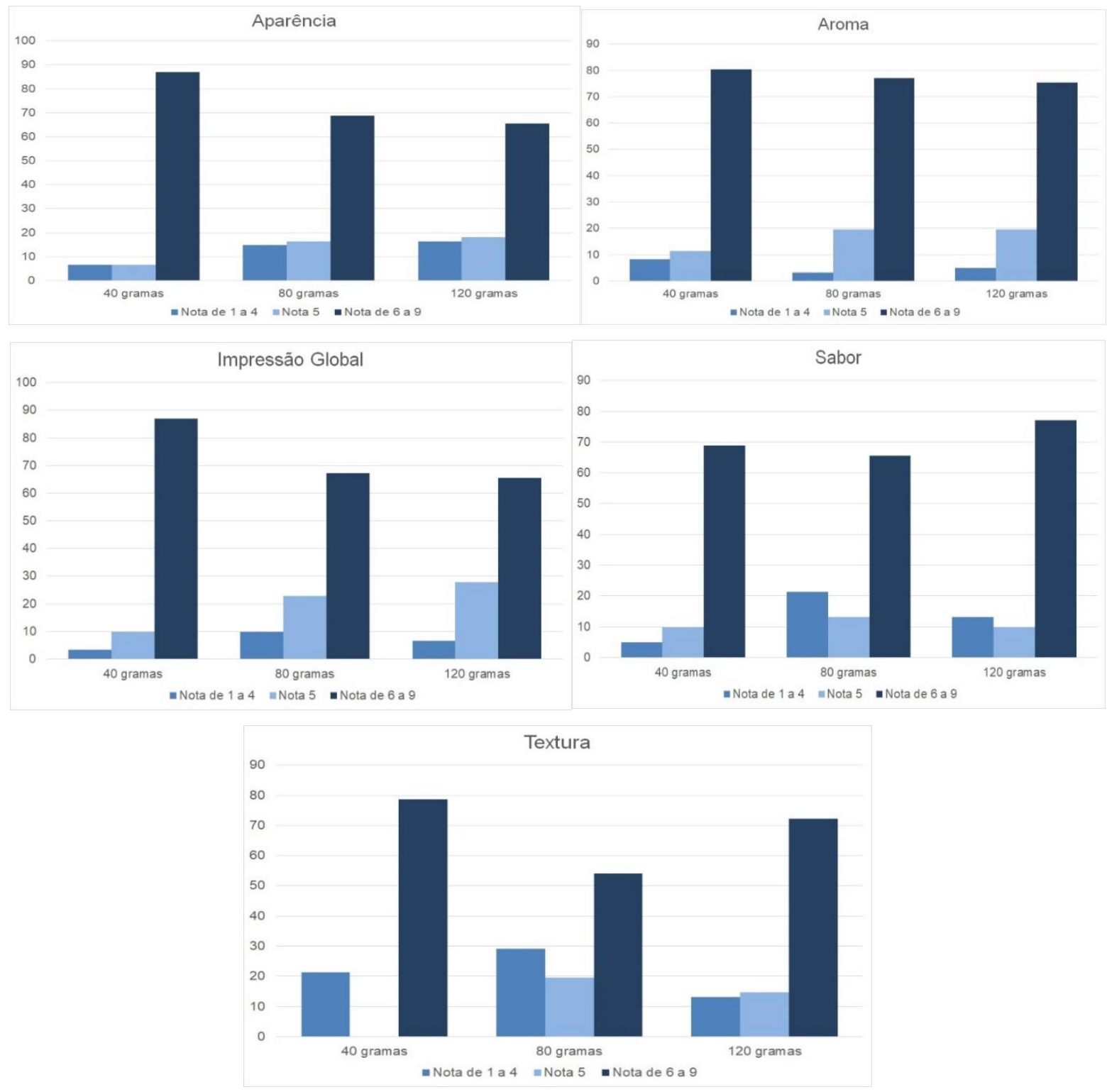

Figura 2. Histograma de frequência quanto à intenção de compra de biscoitos tipo cookie enriquecido com diferentes concentrações de farinha de baru.

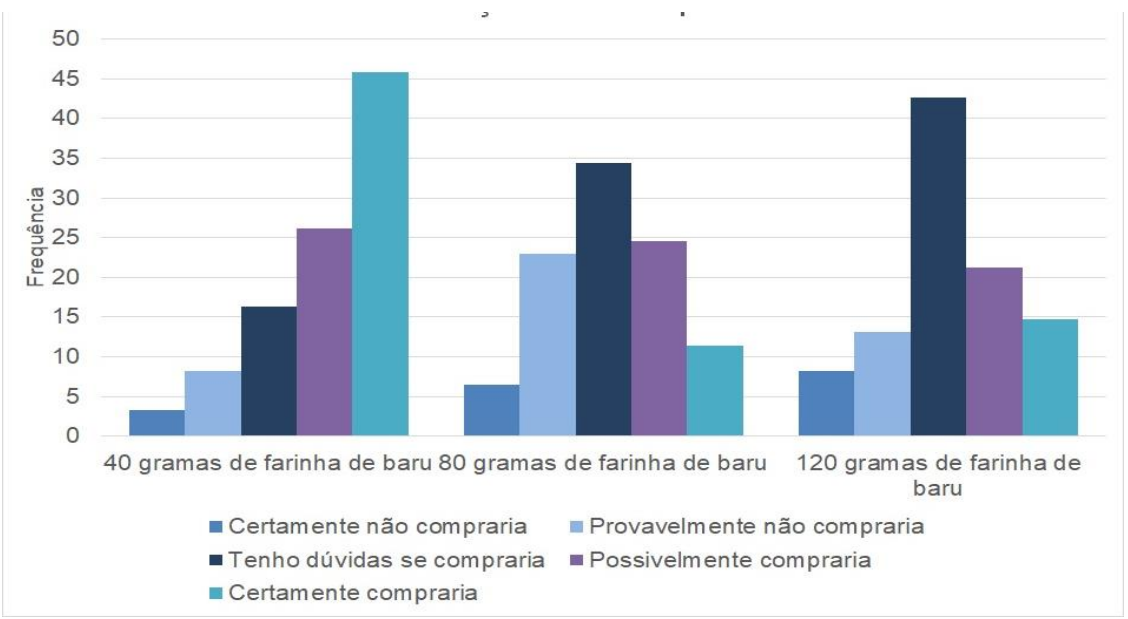


Com relação à intenção de compra (Figura 2 podemos observar que aproximadamente $46 \%$ dos provadores afirmaram que provavelmente e/ou certamente comprariam o cookie com 40 gramas de farinha de baru, $27 \%$ afirmaram que provavelmente e/ou certamente comprariam o mesmo produto enquanto $45 \%$ afirmaram que tinham dúvidas se comprariam o cookie com 120 gramas de farinha de baru. Apenas 4\% dos provadores afirmaram que certamente ou provavelmente não comprariam o cookie enriquecido com 40 gramas de farinha de baru. Este resultado mostra a boa aceitação que a farinha de amaranto juntamente com a farinha de baru teria ao serem ofertadas aos consumidores celíacos.

Quanto à atitude de compra, Queiroz et al. (2017) ao elaborar e caracterizar cookies sem glúten enriquecidos com farinha de coco para celíacos detectaram que os consumidores expressaram uma atitude positiva, caso os cookies enriquecidos com farinha de coco estivessem disponíveis no mercado, declarando que cerca de $54 \%$ consumiriam o produto. $\mathrm{O}$ percentual positivo de intenção de compra foi avaliado pelo somatório das categorias "provavelmente compraria" e "certamente compraria".

Diversas pesquisas têm mostrado que os portadores de doença celíaca têm dificuldade de dar sequência ao tratamento dietético devido à escassez de produtos isentos de glúten. Contudo essa problemática tem diminuído, pois produtos alternativos isentos de glúten estão sendo lançados e se tornando atrativos aos consumidores, como é o caso deste produto que foi bem aceito pelo consumidor e assegura ao celíaco confiança ao consumir um cookie de alto valor proteico formulado com farinha de amaranto e enriquecido com diferentes porções de farinha de baru.

\section{Análise de custos das preparações}

A partir da elaboração das fichas técnicas com os ingredientes listados na Tabela 1, determinou-se o custo de cada formulação, rendimento e custo por porção, como descrito na Tabela 3.

Tabela 3. Ficha técnica das formulações.

\begin{tabular}{cccc}
\hline & \multicolumn{3}{c}{ Formulações } \\
\hline Rendimento total (quantidade) & I & II & III \\
Peso aproximado por porção após cocção $(\mathrm{g})$ & 68 & 68 & 70 \\
Preço de custo da formulação $(\mathrm{R} \$)$ & 10 & 10 & 10 \\
Preço de custo por unidade $(\mathrm{R} \$)$ & 10,32 & 10,72 & 11,12 \\
& 0,15 & 0,16 & 0,16 \\
\hline
\end{tabular}

Foram encontrados cinco tipos de biscoitos tipo cookie sem glúten de marcas variadas encontradas à venda atualmente em casas de produtos naturais e supermercados de
Palmas-TO. O preço unitário encontrado para $10 \mathrm{~g}$ do produto variou entre $\mathrm{R} \$ 0,67$ a $\mathrm{R} \$ 1,33$, e quando comparado aos dados apresentados na Tabela 3 é possível afirmar preliminarmente 
que o produto está dentro do valor de mercado, e possivelmente conseguiria se manter mesmo após o acréscimo de custos inicialmente desconsiderados como os custos com água, gás, energia elétrica, funcionários, impostos, embalagens e depreciação dos equipamentos, entre outros.

\section{Composição nutricional}

A Quadro 1 apresenta a composição nutricional calculada quantitativamente a partir da base de dados da Tabela Brasileira de Composição de Alimentos - TACO. Para calcular o valor energético total das formulações utilizou-se os seguintes fatores de conversão: Carboidratos: 4 kcal.g ${ }^{-1}$; Proteínas: 4 kcal.g ${ }^{-1}$ e Lipídeos por 9 kcal.g ${ }^{-1}$.

Marcelino et al (2017) utilizaram farinha de casca e polpa de baru na elaboração de cupcakes, e os valores diários (VD) encontrados em porção de 40 gramas (4 unidades) para carboidratos foi de 18,94\%, proteínas de 9,4\% e gorduras totais de $17,74 \%$, valores acima do encontrado no estudo.

Cabrera-Chavez (2012) destacou em seu estudo que o enriquecimento de alimentos com a farinha de amaranto pode elevar de $30-40 \%$ a quantia de proteínas no produto, o que é ideal para produtos destinados ao público celíaco, visto que deficiências de nutrientes são comuns em pacientes com diagnóstico de doença celíaca.
Quadro 1. Composição nutricional dos biscoitos elaborados com diferentes proporções de farinha de baru.

a) Biscoito com $40 \mathrm{~g} \mathrm{(5 \% )} \mathrm{de} \mathrm{farinha} \mathrm{de} \mathrm{baru.}$

\begin{tabular}{|c|c|c|}
\hline \multicolumn{2}{|c|}{ INFORMAÇÃ̃ NUTRICIONAL } \\
\hline \multicolumn{2}{|c|}{ Porção de $30 \mathrm{~g}(3$ unidades $)$} \\
\hline Quantidade por porção & $\% \mathrm{VD}(*)$ \\
\hline Valor Energético & $140 \mathrm{kcal} / 585 \mathrm{~kJ}$ & 7 \\
\hline Carboidratos & $12,0 \mathrm{~g}$ & 4 \\
\hline Proteínas & $2,5 \mathrm{~g}$ & 3 \\
\hline Gorduras Totais & $6,6 \mathrm{~g}$ & 12 \\
\hline Gorduras & $5,0 \mathrm{~g}$ & 22 \\
\hline Saturadas & N.D.** & N.E.*** \\
\hline Gorduras Trans & $1,0 \mathrm{~g}$ & 4 \\
\hline Fibra Alimentar & $45 \mathrm{mg}$ & 2 \\
\hline Sódio & \\
\hline
\end{tabular}

b) Biscoito com $80 \mathrm{~g}(10 \%)$ de farinha de baru

\begin{tabular}{|l|c|c|}
\hline \multicolumn{2}{|c|}{ INFORMAÇÃo NUTRICIONAL } \\
\hline \multicolumn{2}{|c|}{ Porção de 30g (3 unidades) } \\
\hline Quantidade por porção & $\% \mathrm{VD}(*)$ \\
\hline Valor Energético & $143 \mathrm{kcal} / 600 \mathrm{~kJ}$ & 7 \\
\hline Carboidratos & $12,2 \mathrm{~g}$ & 4 \\
\hline Proteínas & $2,8 \mathrm{~g}$ & 4 \\
\hline Gorduras Totais & $6,9 \mathrm{~g}$ & 12 \\
\hline Gorduras & $5,0 \mathrm{~g}$ & 22 \\
\hline Saturadas & N.D.** & N.E.*** \\
\hline Gorduras Trans & $1,1 \mathrm{~g}$ & 4 \\
\hline Fibra Alimentar & $45 \mathrm{mg}$ & 2 \\
\hline Sódio & & \\
\hline
\end{tabular}

c) Biscoito com $120 \mathrm{~g}(15 \%)$ de farinha de baru

\begin{tabular}{|c|c|c|}
\hline \multicolumn{2}{|c|}{ INFORMAÇÃ̃O NUTRICIONAL } \\
\hline \multicolumn{2}{|c|}{ Porção de 30g (3 unidades) } \\
\hline Quantidade por porção & $\%$ VD (*) \\
\hline Valor Energético & $147 \mathrm{kcal} / 615 \mathrm{~kJ}$ & 7 \\
\hline Carboidratos & $11,8 \mathrm{~g}$ & 4 \\
\hline Proteínas & $2,9 \mathrm{~g}$ & 4 \\
\hline Gorduras Totais & $7,0 \mathrm{~g}$ & 13 \\
\hline Gorduras & $5,0 \mathrm{~g}$ & 22 \\
\hline Saturadas & N.D.** & N.E.*** ${ }^{* *}$ \\
\hline Gorduras Trans & $1,3 \mathrm{~g}$ & 5 \\
\hline Fibra Alimentar & $45 \mathrm{mg}$ & 2 \\
\hline Sódio & \\
\hline
\end{tabular}

\%VD (*) Valores Diários de referência com base em uma dieta de $2.000 \mathrm{kcal}$ ou $8400 \mathrm{~kJ}$. Seus valores diários podem ser maiores ou menores dependendo de suas necessidades energéticas.

(ND**) Valores não determinados;

(NE***) Valores não estabelecidos; 


\section{CONCLUSÃO}

O cookie enriquecido com 40 gramas de baru se sobressaiu sobre as demais formulações, sendo que $70 \%$ dos provadores atribuíram notas entre 6 a 9, valores estes correspondendo entre os termos "gostei ligeiramente" e "gostei muitíssimo", sendo que aproximadamente $46 \%$ dos provadores afirmaram que provavelmente e/ou certamente comprariam. O preço de custo por unidade variou de $R \$ 0,15$ - 0,16, sendo possível afirmar preliminarmente que o produto está dentro do valor de mercado. O biscoito sem glúten do tipo cookie desenvolvido com farinha de amaranto e enriquecido com farinha de baru é uma alternativa de consumo para o público celíaco, apresentando um ótimo conteúdo de nutrientes.

Todos os autores declararam não haver qualquer potencial conflito de interesses referente a este artigo.

\section{REFERÊNCIAS}

ARAUJO, H. M. C.; ARAUJO, W. M. C.; BOTELHO, R. B. A.; ZANDONADI, R. P. Doença celíaca, hábitos e práticas alimentares e qualidade de vida. Rev. Nutr. Campinas, v. 23, n. 3, p. 467-474, 2010.

BRASIL. Conselho Nacional de Saúde. Resolução n ${ }^{\circ}$ 466, de 12 de dezembro de 2012. Aprova normas regulamentadoras de pesquisas envolvendo seres humanos. Brasília: Diário Oficial da União, 2013.

BRASIL. Ministério da Saúde. Secretaria de Atenção à Saúde. Portaria $n^{\circ}$ 1149, de 11 de novembro de 2015: aprova o protocolo clínico e diretrizes terapêuticas da doença celíaca. Brasília: Ministério da Saúde, 2015.

CABRERA-CHAVEZ, F.; BARCA, A. M. C.; ISLAS-RUBIO, A. R.; MARTI, A.;
MARENGO, M.; PAGANI, M. A.; BONOMI, F.; IAMETTI, S. Molecular rearrangements in extrusion processes for the production of amaranth-enriched, gluten-free rice pasta.LWT. Food Science and Technology. v. 47, n. 2, p. 421-426, 2012.

CAPRILES, V. D.; COELHO, K.D.; GUERRA-MATIAS, A. C.; ARÊAS J. A. G. Effects of processing methods on amaranth starch digestibility and predicted glycemic index. J Food Sci. v. 73, n. 7, p. 160-164, 2018.

CHAVES, J.B.P.; SPROESSER, R.L. Práticas de laboratório de análise sensorial de alimentos e bebidas. 1a ed. Viçosa: UFV; 2002. 81p.

COSTA, D.M.A.; BORGES, A.S. Avaliação da produção agrícola do amaranto (Amaranthus Hypochondriacus). Holos ano, v. 21, p. 97-111, 2005.

FERREIRA, D.F. Sisvar: a computer statistical analysis system. Ciência e Agrotecnologia, v. 35, p. 1039-1042, 2011.

JUNIOR, M. S. S.; CALIARI, M.; TORRES, M. C. L.; VERA, R.; TEIXEIRA, J. S.; ALVES, L. C. Qualidade de biscoitos formulados com diferentes teores de farinha de amêndoa de Baru (dipteryx alata vog.). Goiânia-GO, Brasil. Agropec Trop. v. 37, n. 1, p. 51-56, 2007.

LIMA, D. M. Tabela brasileira de composição de alimentos - TACO. Campinas: NEPAUNICAMP; 2006.

MARCELINO, G.; COLETA, I. T.; CANDIDO, C. J.; SANTOS, E. F. Caracterização e análise sensorial de cupcakes elaborados com diferentes concentrações de farinha de casca e polpa de baru (Dipteryx alata Vog.) Multitemas, Campo Grande, MS, v. 23, n. 54, p. 265-281, maio/ago. 2018

MARIANI, M.; OLIVEIRA, V. R.; FACCIN, R.; RIOS, A. O.; VENZKE, J. G. Elaboração e avaliação de biscoitos sem glúten a partir de farelo de arroz e farinhas de arroz e de soja. Braz. J. Food Technol. 2015, vol.18, n.1p.70-78.

OLIVEIRA, J. C. S.; SOUZA, V. K. S.; SOUZA, G. S. F.; CORDEIRO, S. A.; SILVA, E. C. A.; SILVA, E. C. A; BARROS, A. L. S.; 
MARTINS, A. C. S. Caracterização FísicoQuímica da Farinha de Amêndoas de Baru (Dipteryx Alata Vog.) para Celíacos: uma Breve Revisão. International Journal of Nutrology. v. 11. n. 1, 2018.

ORTOLAN, A. V.; EING, K. K. C.; SANTOS, M. M. R.; CANDIDO, C. J.; SANTOS, E. F.; NOVELLO, D. Adição de farinha de baru em cupcakes: caracterização físico-química e sensorial entre crianças. O Mundo da Saúde, São Paulo, v. 40, n. 2, p. 213-220, 2016.
QUEIROZ, A. M.; ROCHA, R. F. J.; GARRUTI, D. S.; SILVA, A. P. V.; ARAÚJO, Í. M. S. Elaboração e caracterização de cookies sem glúten enriquecidos com farinha de coco: uma alternativa para celíacos. Braz. J. Food Technol. 2017, v. 20, e2016097, 2017.

SILVA, M. R.; SILVA, M. A.A.P.; CHANG, Y.K. Utilização da farinha de jatobá (Hymenaea stigonocarpa Mart.) na elaboração de biscoitos tipo cookie e avaliação da aceitação por testes sensoriais afetivos uni variados e multivariados. Ciência e Tecnologia de Alimentos, v. 18, p. 25-34, 1998 